\title{
Análise Multivariada das Medidas Morfométricas de Potros da Raça Mangalarga Marchador: Análise Discriminante ${ }^{1}$
}

\author{
Luís Fernando Batista Pintoㄹ, Fernando Queiroz de Almeida ${ }^{3}$, Celia Raquel Quirino4, Grasiele \\ Coelho Cabral ${ }^{2}$, Pedro Cezar Nehme de Azevedo ${ }^{5}$, Edson Mauro Santos ${ }^{6}$
}

\begin{abstract}
RESUMO - Objetivou-se, com este trabalho, avaliar as medidas morfométricas de potros e potras da raça Mangalarga Marchador utilizando a análise discriminante. As medidas corporais foram feitas na semana do nascimento dos potros, em 55 potros e 41 potras; aos seis meses de idade, em 35 potros e 37 potras; e aos doze meses de idade, em 19 potros e 31 potras. Foram efetuadas 25 mensurações lineares, envolvendo altura, comprimento, largura e perímetros além de 11 medidas angulares. A análise discriminante foi utilizada para verificar as diferenças morfológicas entre os animais em função do sexo e das idades dentro de cada sexo. As medidas de relevantes para evidenciar o dimorfismo sexual foram as seguintes: nos animais recém-nascidos, os perímetros do boleto e do joelho, os comprimentos do corpo e da cabeça, a altura na cernelha, a distância da soldra ao jarrete e o ângulo fêmur-tibial; aos seis meses de idade, os perímetros do joelho, do tórax e do boleto, o comprimento do pescoço e o ângulo escápulo-solo; aos doze meses de idade, os perímetros do joelho e do tórax, as alturas na cernelha e na garupa, e os ângulos úmero-radial e coxal-solo. Os machos foram, em geral, maiores que as fêmeas nas medidas lineares exceto o perímetro torácico, cujo valor médio foi maior nas potras. Os erros de classificação quanto à idade foram observados, principalmente, após os quatro meses de idade podendo indicar os efeitos ambientais no período pós-desmame.
\end{abstract}

Palavras-chave: eqüinos, medidas angulares, medidas lineares, morfologia

\section{Multivariate Analysis of Body Measures in Mangalarga Marchador Foals: Discriminant Analysis}

\begin{abstract}
This work aims to evaluate the body measures of colts and fillies of Mangalarga Marchador breed using a discriminant analysis. Measures were performed at birth week, in 55 colts and 41 fillies; at six months age, in 35 colts and 37 fillies; and at twelve months age, in 19 colts and 31 fillies. There were made 25 linear measures, including height, length, width and girth, plus 11 angular measures. Discriminant analysis was used to verify morphologic differences between the animals, in function of sex and age within each sex. In newly born, fetlock and knee girths, body and head lengths, withers height, distance from stifle to hock and femur-tibial angle were the measures that has been differentiated sex. At six months of age, knee girth, thoracic girths and fetlock girth, neck length and shoulder-floor angle were measures that has been differentiated sex. At twelve months of age, knee and thoracic girths, withers and croup heights, humerus-radial and coxal-floor angles had evidenced sex dimorphism. The males were in general larger than females for linear measures, except for the thoracic girth, which was larger in the females. Errors of age classification were observed mainly after fourth months of age which indicate environmental influence after weaning.
\end{abstract}

Key Words: angle measures, horse, linear measures, morphometric

\section{Introdução}

A avaliação do crescimento por intermédio da variação do tamanho corporal: peso, altura, perímetro torácico, entre outros, por unidade de tempo fornece valores que podem ser utilizados não somente para avaliação nutricional, como também para seleção genética. O desenvolvimento de eqüinos jovens, geral- mente, é avaliado pelo peso corporal e por medidas como altura na cernelha, altura na garupa, comprimento do corpo, comprimento da cabeça, perímetro torácico e perímetro da canela, dentre outras (Cabrera et al., 1990; Thompson, 1995; Manso Filho et al., 1998; Almeida et al., 2000; Rezende et al.; 2000b).

Alguns trabalhos científicos têm demonstrado haver diferenças morfométricas entre machos e fê-

\footnotetext{
${ }^{1}$ Parte da Dissertação do primeiro autor apresentada ao Programa de Pós-graduação em Zootecnia da UFRRJ. Projeto de pesquisa financiado pela FAPERJ.

2 Zootecnista. Mestre em Zootecnia - UFRRJ - Seropédica - RJ.

3 Professor Adjunto. Instituto de Veterinária - UFRRJ. Bolsista do CNPq

Correspondência: DMCV-IV-UFRRJ. BR 465, km 7, Seropédica, RJ. 23890-000. E.mail: falmeida@ufrrj.br

${ }^{4}$ Professor Associado - LMGA-CCTA-UENF - Campos dos Goytacazes - RJ. E.mail: crq@uenf.br

5 Professor Adjunto. Departamento de Genética-IB-UFRRJ Seropédica, RJ. 23890-000. E.mail: nehme@ufrj.br

6 Discente de graduação em Zootecnia.- UFRRJ. Bolsista de Iniciação Científica PIBIC/CNPq.
} 
meas de diversas raças de eqüinos. Carneiro et al. (1952) elaboraram um dos primeiros levantamentos dos dados de registro da raça Mangalarga Marchador nos quais foram avaliadas as alturas na cernelha e na garupa, comprimentos do corpo e da cabeça, largura da cabeça e perímetros do tórax e da canela, observando valores médios de altura na cernelha de 1,51 e 1,44 m, nos machos e fêmeas adultas, respectivamente. Para efeito de registro definitivo dos eqüinos na Associação Brasileira de Criadores do Cavalo Mangalarga Marchador - ABCCMM (ABCCMM, 1992), recomenda-se altura na cernelha ideal de 1,52 m, nos machos, admitindo-se os valores mínimo e máximo de 1,47 e 1,57 m, respectivamente, e, nas fêmeas, de 1,46 m, com valores mínimo e máximo de 1,40 e $1,54 \mathrm{~m}$, respectivamente.

Recentemente, Zamborlini et al. (1996), avaliando eqüinos adultos da raça Mangalarga Marchador, constataram diferenças entre os sexos, sendo em geral, os machos mais altos do que as fêmeas, com valores médios superiores na altura na cernelha e na garupa, respectivamente, de 1,50 e 1,59 $\mathrm{m}$, nos machos, e de 1,45 e 1,45 m, nas fêmeas. Os autores verificaram valores médios superiores nos machos para o comprimento do corpo, da cabeça, do pescoço, do dorso, da garupa e da espádua, dos perímetros torácico e da canela e, similares aos das fêmeas para os valores médios da largura da cabeça e da anca.

Entretanto, as características morfométricas apresentam, em muitos casos, alta correlação. Zamborlini et al. (1996) observaram, nos eqüinos adultos da raça Mangalarga Marchador, valores de correlação fenotípica elevados entre as medidas da altura na cernelha e as da altura na garupa e do comprimento do corpo, de 0,93 e 0,70 , respectivamente, e valores de correlação fenotípica moderados, variando de 0,39 a 0,50 , entre as medidas do comprimento do corpo e as medidas de largura da anca, perímetro torácico e comprimentos da cabeça, do pescoço, da garupa e da espádua. Portanto, é necessário avaliar as medidas corporais por meio de análises de natureza multivariada, que consideram a estrutura de correlação entre as diferentes medidas utilizadas.

Segundo Faria (1998), a proposição de índices de seleção para eqüinos aos seis meses de idade é viável, desde que sejam consideradas as medidas de altura na cernelha, comprimento do corpo, perímetro torácico e da canela e do peso corporal, o que possibilitaria o desenvolvimento corporal harmônico e equilibrado nos animais selecionados. Os eqüinos da raça
Mangalarga Marchador são animais de sela que apresentam andamento marchado; outras medidas lineares e também medidas angulares dos raios ósseos das principais articulações envolvidas com a dinâmica do andamento devem ser avaliadas para melhor caracterização do padrão racial e do desenvolvimento dos potros da raça. Portanto, a avaliação do crescimento dos potros Mangalarga Marchador pode ser efetiva na seleção genética e fenotípica de futuros reprodutores.

Objetivou-se, neste trabalho, avaliar eqüinos da raça Mangalarga Marchador em diferentes idades, do nascimento aos doze meses de idade, por meio de medidas corporais, utilizando a análise discriminante para a definição de equações que identifiquem animais sub ou superdesenvolvidos e melhor caracterização fenotípica dos animais quanto ao sexo.

\section{Material e Métodos}

Este trabalho foi desenvolvido na Universidade Federal Rural do Rio de Janeiro, com dados de mensuração de eqüinos da raça Mangalarga Marchador distribuídos em diversos criatórios, nas regiões circunvizinhas ao campus universitário, entre agosto de 2000 e junho de 2003. As medidas corporais foram feitas na semana do nascimento dos animais, em 55 potros e 41 potras; aos seis meses de idade, em 35 potros e 37 potras; e aos doze meses de idade, em 19 potros e 31 potras.

As mensurações foram feitas com os animais em estação forçada, isto é, membros, anterior e posterior, na perpendicular sobre um piso plano, formando um paralelogramo retangular, de forma que, visto de perfil, seus membros, para cada bípede, se encobrem e vistos de frente ou detrás estão na vertical e igualmente apoiados no piso conforme descrito por Torres \& Jardim (1992). As medidas foram obtidas sempre do lado direito do animal utilizando-se um hipômetro, uma fita métrica, um artrogoniômetro e um angulador de casco.

Foram efetuadas as medidas lineares: altura na cernelha, na garupa e do costado; comprimento do corpo, da garupa, do dorso-lombo, da escápula, do pescoço, da cabeça e dos cascos anterior e posterior; largura da cabeça, do peito e da anca; distância da ponta da escápula ao boleto, do codilho ao solo, do codilho ao joelho, do joelho ao boleto, da soldra ao jarrete e do jarrete ao boleto; perímetro torácico, do antebraço, do joelho, do boleto e da canela. Também

\section{R. Bras. Zootec., v.34, n.2, p.600-612, 2005}


foram efetuadas as medidas angulares: escápulosolo, escápulo-umeral, úmero-radial, metacarpofalangeano, coxal-solo, coxofemoral, fêmur-tibial, tíbio-metatarsiano, metatarso-falangeano e casco-solo do membro anterior e posterior.

Os procedimentos de mensuração foram descritos por Camargo \& Chieffi (1971), Toledo (1985), Torres \& Jardim (1992), Nascimento (1999) e Cabral et al. (2004ab), exceto para as medidas das distâncias da ponta da escápula ao boleto, do joelho ao boleto e do jarrete ao boleto, descritas por Thompson (1995), e para as medidas das distâncias do codilho ao joelho e da soldra ao jarrete, descritas por Cabral (2002). As medidas utilizadas foram escolhidas tendo como critério o emprego das mesmas na avaliação morfológica de eqüinos da raça Mangalarga Marchador (Barbosa, 1993; Zamborlini, 1996; Nascimento, 1999), bem como a necessidade de disponibilizar informações sobre medidas angulares e novas medidas lineares ainda não consideradas em outros estudos.

A análise discriminante foi utilizada com o propósito de obter equações para classificação dos animais de acordo com o sexo e idade, tomando como base as medidas lineares e angulares. Na discriminação das idades, foram utilizados animais ao nascimento e aos 2 , $4,6,8,10$ e 12 meses de idade, enquanto, na discriminação dos sexos, foram utilizados animais recémnascidos e aos 6 e 12 meses de idade.

A metodologia de análise discriminante baseia-se na obtenção de equações do tipo: $Y=\beta_{0}+\beta_{1} X_{1}+\beta_{2} x_{2}+\ldots+\beta_{i} X_{i}$, em que $\beta_{0}$ é uma constante; $\beta_{i}$ referese-se aos coeficientes de ponderação associados a $X_{i}$ variáveis da equação; e $Y$ é um índice obtido para o referido objeto na análise. Segundo Khattree \& Naik (2000), os $\beta_{i}$ coeficientes são obtidos de acordo com algum critério de agrupamento; neste trabalho, esses critérios foram o sexo e a idade, separadamente. Após a obtenção dos coeficientes de ponderação, foi possível reagrupar os animais de acordo com as equações; a equação que forneceu o maior índice determinou o grupo em que o animal foi inserido.

Foram utilizados os procedimentos DISCRIM e STEPDICS do programa SAS - Statistical Analysis System (SAS, 1996). O procedimento DISCRIM teve por finalidade gerar as funções e classificar os animais, enquanto o procedimento STEPDISC identificou as variáveis importantes no processo de discriminação dos grupos por faixa etária e por sexo.

\footnotetext{
R. Bras. Zootec., v.34, n.2, p.600-612, 2005
}

\section{Resultados e Discussão}

Nos animais recém-nascidos, as medidas de perímetro do boleto, comprimento do corpo, comprimento da cabeça, altura na cernelha, distância da soldra ao jarrete e perímetro do joelho possibilitaram discriminar os potros das potras (Tabela 1).

Os potros apresentaram valores médios maiores que as potras em todas as seis medidas, o que está de acordo com o observado por Saastamoinen (1990) avaliando potros recém-nascidos da raça Finnhorse. No entanto, Green (1969), avaliando o crescimento de potros da raça Puro Sangue Inglês (PSI), do nascimento aos 12 meses de idade, não observou diferença entre os sexos para a medida de altura na cernelha, o que pode ser uma característica da raça Puro Sangue Inglês ou o resultado da utilização de testes univariados na comparação das médias, os quais apresentam menor capacidade de avaliação das diferenças entre os valores que as análises multivariadas nas situações em que as características apresentam correlação significativa.

Vários fatores interferem no crescimento prénatal dos animais sendo estes o tamanho materno, o número de partos e a nutrição da matriz, tamanho da placenta e estresse climático e, destes, o mais importante é o tamanho materno (Jainudee \& Hafez, 1982). Segundo Breier \& Gluckman (1991), o crescimento fetal está adaptado às condições maternas e/ou às limitações nutricionais via placenta e, nos períodos pré e pós-parto, o eixo hormonal somatotrópico passa por alterações relacionadas ao padrão de secreção do Hormônio do Crescimento - GH, no número de receptores hepáticos e nos níveis plasmáticos dos Fatores de Crescimento semelhantes à Insulina, IGF-I e IGF-II.

Considerando o padrão de secreção do GH nos animais recém-nascidos, Breier et al. (2000) observaram a ocorrência de dimorfismo sexual na secreção de GH em várias espécies, com pulsos elevados de secreção hormonal e níveis basais menores nos machos que nas fêmeas e a possível relação entre o padrão de secreção do GH e as diferenças na secreção do Fator Liberador do GH, no sistema portahipofisário dos machos e fêmeas.

Os coeficientes de variação das medidas do perímetro do boleto, comprimento do corpo, comprimento da cabeça, altura na cernelha, distância da soldra ao jarrete perímetro do joelho podem ser considerados baixos, haja vista a dificuldade de 
Tabela 1- Valores médios, desvios-padrão (DP), coeficientes de variação (CV) e parâmetros da função discriminante $\left(\beta_{\mathrm{s}}\right)$ de potros e potras recém-nascidos, obtidos das medidas lineares

Table 1 - Average, standard deviations (DP), coefficients of variation (CV) and parameters of discriminant function ( $\beta_{s}$ ) of colts and fillies newly born, obtained from linear measures

\begin{tabular}{|c|c|c|c|c|c|c|c|c|}
\hline \multirow[t]{2}{*}{$\begin{array}{l}\text { Variável } \\
\text { Variable }\end{array}$} & \multicolumn{4}{|c|}{$\begin{array}{c}\text { Potros } \\
\text { Colts }\end{array}$} & \multicolumn{4}{|c|}{$\begin{array}{l}\text { Potras } \\
\text { Fillies }\end{array}$} \\
\hline & $\beta_{\mathrm{s}}$ & $\begin{array}{c}\text { Média }(\mathrm{cm}) \\
\text { Average }\end{array}$ & $\mathrm{DP}(\mathrm{cm})$ & $\mathrm{CV} \%$ & $\beta_{\mathrm{s}}$ & $\begin{array}{c}\text { Média }(\mathrm{cm}) \\
\text { Average }\end{array}$ & $\mathrm{DP}(\mathrm{cm})$ & CV\% \\
\hline$\overline{\mathrm{PB}}$ & 7,90351 & 18,39 & $\pm 0,8$ & 4,37 & 6,31892 & 17,5 & $\pm 0,85$ & 4,87 \\
\hline $\mathrm{CC}$ & $-0,96705$ & 68,15 & $\pm 3,8$ & 5,58 & $-0,71274$ & 68,02 & $\pm 4,64$ & 6,82 \\
\hline Ccab & 3,93632 & 32,2 & $\pm 1,84$ & 5,71 & 3,57822 & 31,17 & $\pm 2,35$ & 7,55 \\
\hline $\mathrm{AC}$ & 0,32356 & 93,4 & $\pm 4,14$ & 4,43 & 0,60915 & 92,85 & $\pm 4,49$ & 4,84 \\
\hline DSJ & 4,14774 & 34,87 & $\pm 2,54$ & 7,28 & 3,89884 & 33,66 & $\pm 2,67$ & 7,93 \\
\hline PJ & 18,17702 & 21,55 & $\pm 0,82$ & 3,8 & 17,17829 & 20,9 & $\pm 0,95$ & 4,55 \\
\hline $\begin{array}{l}\text { Constante }\left(\beta_{0}\right) \\
\text { Constant }\end{array}$ & $-386,906$ & - & - & - & $-361,573$ & - & - & - \\
\hline
\end{tabular}

PB - perímetro do boleto; CC - comprimento do corpo; Ccab - comprimento da cabeça; AC - altura na cernelha; DSJ - distância da soldra ao jarrete; PJ - perímetro do joelho.

PB - fetlock girth; CC - body lenght; Ccab - head length; AC - Withers heigth; DSJ - distance between stifle and hock; PJ - knee girth.

mensurar os animais recém-nascidos, e estão de acordo com os resultados observados por Saastamoinen (1990) em potros Finnhorse, inferiores a $10 \%$. Os valores observados são próximos dos descritos por García et al. (1987), avaliando medidas de altura, comprimento e perímetros em eqüinos adultos da raça Árabe cuja medida de maior coeficiente de variação, tanto nos machos quanto nas fêmeas, foi a largura do costado, de 6,91 e 9,28, respectivamente. Molina et al. (1999), por sua vez, observaram que o perímetro do joelho, com coeficiente de variação igual a $5,1 \%$, foi a medida de maior variação em eqüinos adultos da raça Andaluz, valor um pouco acima do observado no presente trabalho.

Os valores correspondentes aos coeficientes de ponderação $\left(b_{\mathrm{s}}\right)$ das respectivas medidas lineares e a constante $\left(b_{0}\right)$ podem ser utilizados na formulação da função discriminante. A partir dos valores obtidos das mensurações dos animais para as medidas do perímetro do boleto, comprimento do corpo, comprimento da cabeça, altura na cernelha, distância da soldra ao jarrete e perímetro do joelho, é possível calcular dois índices para cada animal mensurado, cada índice utilizando uma destas funções. O maior índice determinou em qual grupo o animal devia ser incluído e, desta forma, todos os animais foram classificados de acordo com o sexo. Estas equações podem ser posteriormente utilizadas na avaliação de outros animais recémnascidos da raça Mangalarga Marchador, para verificar se ao nascimento os animais apresentam padrão fenotípico correspondente ao sexo.
A nova classificação pode ser observada na Tabela 2. Das 41 potras recém-nascidas, oito foram classificadas como potros e dos 55 potros recémnascidos, nove foram classificados como potras, o que correspondeu a percentuais de erros de $19,5 \% \mathrm{e}$ $16,4 \%$, nas potras e potros, respectivamente, e acerto médio de $82,1 \%$.

A análise discriminante forneceu os parâmetros que permitem elaborar as equações, de acordo com o sexo, para classificar os potros e potras de seis meses de idade, em machos e fêmeas como pode ser observado na Tabela 3 .

Os perímetros do joelho, torácico e do boleto mais o comprimento do pescoço possibilitaram discriminar os potros das potras, nesta idade, ocorrendo redução no número de medidas capazes de diferenciar os dois sexos do nascimento para o sexto mês, e duas novas

Tabela 2 - Classificação de potras e potros recém-nascidos, em função das medidas lineares

Table 2 - Classification of fillies and colts newly born, in function of linear measures

\begin{tabular}{ccc}
\hline Grupo observado & \multicolumn{2}{c}{ Após a classificação } \\
Observed group & \multicolumn{2}{c}{ Afterclassification } \\
\cline { 2 - 3 } & Potras & Potros \\
& Fillies & Colts \\
\hline Potras (Fillies) & 33 & 9 \\
$\%$ & 80,5 & 16,4 \\
Potros (Colts) & 8 & 46 \\
$\%$ & 19,5 & 83,6 \\
Total & 41 & 55 \\
\hline
\end{tabular}

Percentual de acertos $=82,1 \%$

Correctness percentage $=82.1 \%$ 
Tabela 3 - Valores médios, desvios-padrão (DP), coeficientes de variação (CV) e parâmetros da função discriminante $\left(\beta_{\mathrm{s}}\right)$ de potros e potras com seis meses de idade, obtidos das medidas lineares

Table 3 - Average, standard deviation (DP), variation coefficients (CV) and parameters of discriminant function ( $\beta_{s}$ ) of colts and fillies with six months age, obtained from linear measures

\begin{tabular}{|c|c|c|c|c|c|c|c|c|}
\hline \multirow[t]{2}{*}{$\begin{array}{l}\text { Variável } \\
\text { Variable }\end{array}$} & \multicolumn{4}{|c|}{$\begin{array}{l}\text { Potro } \\
\text { Colt }\end{array}$} & \multicolumn{4}{|c|}{$\begin{array}{l}\text { Potra } \\
\text { Filly }\end{array}$} \\
\hline & $\beta_{\mathrm{s}}$ & $\begin{array}{c}\text { Média }(\mathrm{cm}) \\
\text { Average }\end{array}$ & $\mathrm{DP}(\mathrm{cm})$ & $\mathrm{CV} \%$ & $\beta_{\mathrm{s}}$ & $\begin{array}{c}\text { Média }(\mathrm{cm}) \\
\text { Average }\end{array}$ & $\mathrm{DP}(\mathrm{cm})$ & $\mathrm{CV} \%$ \\
\hline$\overline{\mathrm{CP}}$ & 2,77023 & 51,54 & $\pm 2,7$ & 5,25 & 2,57189 & 50,38 & $\pm 2,58$ & 5,11 \\
\hline PT & 1,72048 & 128,6 & $\pm 4,11$ & 3,20 & 2,08548 & 129,35 & $\pm 4,56$ & 3,52 \\
\hline PJ & 46,49795 & 26,73 & $\pm 0,71$ & 2,66 & 43,82523 & 25,81 & $\pm 0,63$ & 2,43 \\
\hline PB & 7,19131 & 22,71 & $\pm 0,95$ & 4,18 & 6,51286 & 21,95 & $\pm 0,86$ & 3,94 \\
\hline $\begin{array}{l}\text { Constante }\left(\begin{array}{ll}\beta & 0\end{array}\right) \\
\text { Constant }\end{array}$ & $-885,825$ & - & - & - & $-837,376$ & - & - & - \\
\hline
\end{tabular}

CP - Comprimento do pescoço; PT - Perímetro torácico; PJ - Perímetro do joelho; PB - Perímetro do boleto.

$C P$ - neck length; $P T$ - chest girth; $P J$ - knee girth; $P B$ - fetlock girth.

medidas podem ser observadas, o perímetro torácico e o comprimento do pescoço.

Os machos foram maiores que as fêmeas paras as medidas dos perímetros do joelho e do boleto e comprimento do pescoço e menores no perímetro torácico, em decorrência, provavelmente, da seleção nas fêmeas para maior profundidade do corpo e arqueamento de costelas. Árnason (1984) observou que éguas da raça Icelandic Toelter apresentaram, em média, valores maiores do perímetro torácicos do que garanhões, entretanto, Zamborlini et al. (1996) verificaram que garanhões da raça Mangalarga Marchador apresentaram valores de perímetro torácico superiores aos das éguas. Os coeficientes de variação destas medidas foram menores que $10 \%$ e semelhantes aos observados por Saastamoinen (1990), em potros da raça Finnhorse, na mesma faixa etária.

$\mathrm{Na}$ Tabela 4 pode ser observada a classificação dos potros e potras com seis meses de idade, obtida com as medidas lineares. Nesta idade, cinco das 37 potras foram classificadas como potros, enquanto cinco dos 35 potros foram classificados como potras, correspondendo a percentuais de erro de $13,5 \%$ e $14,3 \%$, nas potras e potros, respectivamente, e um acerto médio de $86,1 \%$. Portanto, novamente foi possível identificar os animais que estão fora do padrão fenotípico apresentado pelos grupos de machos e fêmeas avaliados.

As funções discriminantes para animais aos 12 meses de idade podem ser elaboradas com os parâmetros contidos na Tabela 5. Como mencionado para as duas idades anteriores, estas funções poderiam ser utilizadas na identificação de animais que estejam fora do padrão fenotípico em relação ao sexo, possibilitando o descarte antecipado daqueles
Tabela 4 - Classificação de potras e potros com seis meses de idade, em função das medidas lineares

Table 4 - Classification of fillies and colts of six months age in function of linear measures

\begin{tabular}{ccc}
\hline Grupo observado & \multicolumn{2}{c}{ Após a classificação } \\
Observed group & \multicolumn{2}{c}{ Afterclassification } \\
\cline { 2 - 3 } & Potras & Potros \\
Fillies & Colts \\
\hline Potras (Fillies) & 32 & 5 \\
$\%$ & 86,5 & 14,3 \\
Potros (Colts) & 5 & 30 \\
$\%$ & 13,5 & 85,7 \\
Total & 37 & 35 \\
\hline
\end{tabular}

Percentual de acertos $=86,1 \%$.

Correctness percentage $=86.1 \%$.

que poderiam não obter o registro definitivo na avaliação morfológica feita pela ABCCMM.

As medidas do perímetro do joelho, perímetro torácico, altura na cernelha e altura na garupa foram as que apresentaram poder de discriminar potros de potras, aos 12 meses de idade. Os potros foram maiores, exceto na medida do perímetro torácico que apresentou valor médio superior nas potras. Segundo Gatford et al. (1998), nos ruminantes e em outras espécies, os níveis plasmáticos de IGF-I no período do desmane são maiores nos machos que nas fêmeas - dimorfismo sexual que é mantido até a puberdade. Breier et al. (2000) reportaram a ocorrência de dimorfismo sexual na secreção do GH e que hormônios esteróides gonadais aumentam, nos ruminantes, a síntese e a secreção de GH na hipósife.

Outro aspecto a ser considerado quanto ao dimorfismo sexual dos animais aos 12 meses de idade é o fato de os machos serem selecionados para maiores alturas que as fêmeas, para efeito de registro 
Tabela 5 - Valores médios, desvios-padrão (DP), coeficiente de variação (CV) e parâmetros da função discriminante $\left(\beta_{\mathrm{s}}\right)$ de potros e potras com doze meses de idade, obtidos das medidas lineares

Table 5 - Average, standard deviations (DP), coefficients of variation (CV) and parameters of discriminant function ( $\beta_{s}$ ) of colts and fillies with twelve months age, obtained from linear measures

\begin{tabular}{|c|c|c|c|c|c|c|c|c|}
\hline \multirow[t]{2}{*}{$\begin{array}{l}\text { Variável } \\
\text { Variable }\end{array}$} & \multicolumn{4}{|c|}{$\begin{array}{c}\text { Potro } \\
\text { Colt }\end{array}$} & \multicolumn{4}{|c|}{$\begin{array}{l}\text { Potra } \\
\text { Filly }\end{array}$} \\
\hline & $\beta_{\mathrm{s}}$ & $\begin{array}{c}\text { Média }(\mathrm{cm}) \\
\text { Average }\end{array}$ & $\mathrm{DP}(\mathrm{cm})$ & $\mathrm{CV} \%$ & $\beta_{\mathrm{s}}$ & $\begin{array}{c}\text { Média }(\mathrm{cm}) \\
\text { Average }\end{array}$ & $\mathrm{DP}(\mathrm{cm})$ & $\mathrm{CV} \%$ \\
\hline$\overline{\mathrm{PJ}}$ & 27,55376 & 28,26 & $\pm 0,81$ & 2,85 & 23,92185 & 27,23 & $\pm 0,92$ & 3,38 \\
\hline PT & $-5,29851$ & 143,21 & $\pm 5,04$ & 3,52 & $-4,72617$ & 145,42 & $\pm 7,01$ & 4,82 \\
\hline $\mathrm{AC}$ & 8,96118 & 133,32 & $\pm 3,33$ & 2,50 & 7,26373 & 132,10 & $\pm 4,02$ & 3,04 \\
\hline AG & 4,19643 & 133,63 & $\pm 3,25$ & 2,43 & 5,65275 & 133,35 & $\pm 3,83$ & 2,87 \\
\hline Constante $(\beta 0)$ & $-888,666$ & - & - & - & $-839,154$ & - & - & - \\
\hline
\end{tabular}

PJ - perímetro do joelho; PT - perímetro torácico; AC - altura na cernelha; AG - altura na garupa.

$P J$ - knee girth; PT - toracic girth; $A C$ - withers heigth; $A G$ - croup heigth.

definitivo, aos 36 meses de idade, na ABCCMM, e à moderada estimativa de herdabilidade da altura na cernelha, de 0,49, observada por Zamborlini et al. (1996), em eqüinos da raça Mangalarga Marchador.

Os coeficientes de variação permaneceram baixos, como nas faixas etárias inferiores, e semelhantes aos obtidos por Saastamoinen (1990), em potros Finnhorse.

Nesta idade, observou-se que uma das 31 potras foi classificada como potro e nenhum dos machos foi classificado como fêmea, o que correspondeu a um acerto médio de 98,0\% (Tabela 6). Portanto, aos 12 meses de idade, as diferenças entre machos e fêmeas são bem mais acentuadas que nos animais recémnascidos e de seis meses de idade.

Em relação à análise discriminante das medidas angulares dos animais recém-nascidos, a angulação fêmur-tibial foi a única que possibilitou discriminar os potros das potras recém-nascidas (Tabela 7). Os coeficientes de variação das medidas angulares geralmente foram um pouco maiores que os obtidos com medidas lineares, o que provavelmente é decorrente da maior dificuldade de obtenção dos ângulos, principalmente em animais recém-nascidos.

Holmström et al. (1990) observaram, nas mensurações das angulações ósseas de eqüinos adultos da raça Swedish Warmblood, coeficientes de variação entre 2 e $6 \%$, exceto para o ângulo coxal-solo cujo coeficiente de variação foi acima de $10 \%$.

Das 41 potras recém-nascidas, 12 foram classificadas como potros e dos 55 potros recém-nascidos, 21 como potras, correspondendo a percentuais de erro de 29,3 e $38,2 \%$, nas potras e potros, respectivamente (Tabela 8).

R. Bras. Zootec., v.34, n.2, p.600-612, 2005
Tabela 6 - Classificação de potras e potros com doze meses de idade, em função das medidas lineares

Table 6 - Classification of fillies and colts of twelve months age, in function of linear measures

\begin{tabular}{ccc}
\hline $\begin{array}{c}\text { Grupo observado } \\
\text { Observed group }\end{array}$ & \multicolumn{2}{c}{ Após a classificação } \\
& \multicolumn{2}{c}{ Afterclassification } \\
\cline { 2 - 3 } & Potra & Potro \\
Filly & Colt \\
\hline Potra (Filly) & 30 & 0 \\
$\%$ & 96,8 & 0,0 \\
Potro (Colt) & 1 & 19 \\
$\%$ & 3,2 & 100,0 \\
Total & 31 & 19 \\
\hline
\end{tabular}

Percentual de acertos $=98,0 \%$.

Correctness percentage $=98.0 \%$.

Estes percentuais de erro permitiram um acerto médio de $66,3 \%$, inferior ao observado com as medidas lineares, de $82,1 \%$, nesta idade. Logo, com as medidas angulares, não foi possível discriminar claramente os grupos de machos e fêmeas, e, portanto, as medidas lineares são mais indicadas que as angulares na avaliação de animais recém-nascidos quanto ao padrão fenotípico de cada sexo.

Aos seis meses de idade, a angulação escápulosolo foi a única medida que possibilitou discriminar os potros das potras (Tabela 9). O valor médio desta angulação, de $62^{\circ}$, está dentro da amplitude observada por Lage (2001), em eqüinos adultos da raça Mangalarga Marchador, com valores variando entre $52^{\circ}$ e $70^{\circ}$ e próximo do valor médio observado para angulação escápulo-solo, de $66,8^{\circ} \mathrm{em}$ eqüinos adultos da raça Mangalarga Marchador (Cabral et al., 2004a). Entretanto, os valores médios da angulação escápulosolo, tanto de machos quanto de fêmeas, estão acima 
Tabela 7- Valores médios, desvios-padrão (DP), coeficientes de variação (CV) e parâmetros da função discriminante $\left(\beta_{\mathrm{s}}\right)$ de potros e potras recém-nascidos, obtidos das medidas angulares

Table 7 - Average, standard deviations (DP), coefficients of variation (CV) and parameters of discriminant function ( $\left.\beta_{s}\right)$ of colts and fillies newly born, obtained from angular measures

\begin{tabular}{|c|c|c|c|c|c|c|c|c|}
\hline \multirow[t]{2}{*}{$\begin{array}{l}\text { Variável } \\
\text { Variable }\end{array}$} & \multicolumn{4}{|c|}{$\begin{array}{c}\text { Potro } \\
\text { Colt }\end{array}$} & \multicolumn{4}{|c|}{$\begin{array}{l}\text { Potra } \\
\text { Filly }\end{array}$} \\
\hline & $\beta_{\mathrm{s}}$ & $\begin{array}{l}\text { Média }(\mathrm{cm}) \\
\text { Average }\end{array}$ & $\mathrm{DP}(\mathrm{cm})$ & $\mathrm{CV} \%$ & $\beta_{\mathrm{s}}$ & $\begin{array}{c}\text { Média }(\mathrm{cm}) \\
\text { Average }\end{array}$ & $\mathrm{DP}(\mathrm{cm})$ & CV\% \\
\hline AFT & 1,33162 & $101,13^{\circ}$ & $9,43^{\circ}$ & 9,33 & 1,37864 & $106,02^{\circ}$ & $7,2^{\circ}$ & 6,79 \\
\hline $\begin{array}{l}\text { Constante }\left(\begin{array}{l}\beta \\
\text { Constant }\end{array}\right. \\
\text { Cont }\end{array}$ & $-67,816$ & - & - & - & $-72,944$ & - & - & - \\
\hline
\end{tabular}

AFT - ângulo fêmur-tibial.

AFT - femur-tibia angle

Tabela 8 - Classificação de potras e potros recémnascidos, em função das medidas angulares

Table 8 - Classification of fillies and colts newly born, in function of the angular measures

\begin{tabular}{ccc}
\hline Grupo observado & \multicolumn{2}{c}{ Após a classificação } \\
Observed group & \multicolumn{2}{c}{ Afterclassification } \\
\cline { 2 - 3 } & Potra & Potro \\
& Filly & Colt \\
\hline Potra (Filly) & 29 & 21 \\
$\%$ & 70,7 & 38,2 \\
Potro (Colt) & 12 & 34 \\
$\%$ & 29,3 & 61,8 \\
Total & 41 & 55 \\
\hline
\end{tabular}

Percentual de acertos $=66,3 \%$.

Correctness percentage $=66.3 \%$.

das indicações de Andrade (1986) para eqüinos adultos das raças marchadoras, com valores variando de 50 e $55^{\circ}$.

Em eqüinos das raças de andamento ao trote, os valores da angulação escápulo-solo observados nos animais adultos são similares aos dos animais de andamento marchado. Holmstrõm et al. (1990) avaliando eqüinos da raça Swedish Warmblood observaram valores variando de $64,4^{\circ}$ a $66,3^{\circ}$, de acordo com as diferentes modalidades de equitação, ao passo que Zechner et al. (2001), em eqüinos da raça Lipizzaner, observaram valores variando de $57,6^{\circ}$ a $60,9^{\circ}$, para a angulação da escápula com o solo. Os valores acima citados são um pouco maiores que os indicados por Nascimento (1999), de 52 a $58^{\circ}$, para os eqüinos.

Entre as 36 potras com seis meses de idade, 15 foram classificadas como potros, enquanto $13 \mathrm{dos} 34$ potros foram classificados como potras, correspondendo a percentuais de erro de 41,7 e $38,2 \%$, nas potras e potros, respectivamente (Tabela 10). Estes erros permitiram acerto médio de $60,5 \%$, valor próximo ao observado nos animais recém-nascidos e inferior ao observado com as medidas lineares, de $86,1 \%$ nos animais aos seis meses de idade. Novamente, as medidas lineares foram mais eficientes em determinar os grupos de machos e fêmeas e identificar animais fora do padrão fenotípico de cada sexo.

Os ângulos úmero-radial e coxal-solo foram as medidas angulares que permitiram discriminar os machos das fêmeas aos 12 meses de idade (Tabela 11). O ângulo úmero-radial apresentou, em média, valor maior nos potros que nas potras e, segundo Ribeiro (1988), este ângulo apresenta abertura do tipo anterior e é de grande importância para a ação do andamento, o que permite classificá-lo como ângulo impulsor.

Os potros apresentaram, em média, menor angulação de coxal-solo que as potras, na idade de doze meses, de $24,0^{\circ}$ e $25,1^{\circ}$, respectivamente. Em eqüinos adultos da raça Mangalarga Marchador, Cabral et al. (2004a) verificaram valores da angulação de coxal-solo de $19,7^{\circ}$ e $25,5^{\circ}$, nos garanhões e éguas, respectivamente, enquanto Nascimento (1999) indicou valores de $30^{\circ}$ a $35^{\circ}$ da angulação coxal-solo, em equiinos adultos marchadores. Os valores médios do ângulo coxal-solo foram menores que os observados por Lage (2001), de 53, $8^{\circ}$, em animais adultos da raça Mangalarga Marchador e a diferença pode estar relacionada a metodologia utilizada no procedimento de mensuração e a idade dos animais. Segundo Pinto (2003), a medida de maior variação na avaliação morfométrica de eqüinos Mangalarga Marchador foi a do ângulo coxal-solo, com coeficiente de variação de $19,9,15,3,12,1$ e $16,8 \%$, nas potras, e de 21,$8 ; 15,2$; 10,4 e $18,2 \%$, nos potros, ao nascimento, aos 6 e 12 meses de idade e nos animais adultos, respectivamente.

Os valores médios do ângulo coxal-solo estão próximos dos observados por Holmstrõm et al. (1990), em eqüinos adultos da raça Swedish Warmblood, de $29,5^{\circ}$ a $32,5^{\circ}$, enquanto Zechner et al. (2001) observa- 
Tabela 9 - Valores médios, desvios-padrão (DP), coeficientes de variação (CV) e parâmetros da função discriminante $\left(\beta_{\mathrm{s}}\right)$ de potros e potras com seis meses de idade, obtidos das medidas angulares

Table 9 - Average, standard deviations (DP), coefficients of variation (CV) and parameters of discriminant function ( $\beta_{s}$ ) of colts and fillies with six months age, obtained from angular measures

\begin{tabular}{|c|c|c|c|c|c|c|c|c|}
\hline \multirow[t]{2}{*}{$\begin{array}{l}\text { Variável } \\
\text { Variable }\end{array}$} & \multicolumn{4}{|c|}{$\begin{array}{c}\text { Potro } \\
\text { Colt }\end{array}$} & \multicolumn{4}{|c|}{$\begin{array}{l}\text { Potra } \\
\text { Filly }\end{array}$} \\
\hline & $\beta_{\mathrm{s}}$ & $\begin{array}{c}\text { Média }(\mathrm{cm}) \\
\text { Average }\end{array}$ & $\mathrm{DP}(\mathrm{cm})$ & $\mathrm{CV} \%$ & $\beta_{\mathrm{s}}$ & $\begin{array}{c}\text { Média }(\mathrm{cm}) \\
\text { Average }\end{array}$ & $\mathrm{DP}(\mathrm{cm})$ & CV\% \\
\hline AES & 12,74540 & $62,44^{\circ}$ & $\pm 2,11^{\circ}$ & 3,37 & 12,58520 & $61,67^{\circ}$ & $\pm 2,23^{\circ}$ & 3,62 \\
\hline $\begin{array}{l}\text { Constante }\left(\begin{array}{l}\beta \\
\beta\end{array}\right) \\
\text { Constant }\end{array}$ & $-399,288$ & - & - & - & $-389,276$ & - & - & - \\
\hline
\end{tabular}

AES - ângulo escápulo-solo.

AES - shoulder-floor angle.

Tabela 10 - Classificação de potras e potros com seis meses de idade, em função das medidas angulares.

Table 10 - Classification of fillies and colts of six months age, in function of angular measures

\begin{tabular}{ccc}
\hline Grupo observado & \multicolumn{2}{c}{ Após a classificação } \\
Observed group & \multicolumn{2}{c}{ Afterclassification } \\
\cline { 2 - 3 } & Potra & Potro \\
& Filly & Colt \\
\hline Potra (Filly) & 21 & 13 \\
$\%$ & 58,3 & 38,2 \\
Potro (Colt) & 15 & 21 \\
$\%$ & 41,7 & 61,8 \\
Total & 36 & 34 \\
\hline
\end{tabular}

Percentual de acertos $=60.5 \%$

Correctness percentage $=60.5 \%$ ram valores médios variando de $10,9^{\circ}$ a $16,3^{\circ}$ para inclinação da garupa, em éguas da raça Lipizzaner.

Das 31 potras avaliadas, oito foram classificadas como potros, ao passo que dos 19 potros avaliados sete foram classificados como potras, o que correspondeu a percentuais de erros de 25,8 e 36,8 nas potras e potros, respectivamente (Tabela 12). Estes erros permitiram acerto médio de $68,7 \%$, mas estão bem abaixo dos resultados obtidos com as medidas lineares nos animais aos 12 meses de idade.

Os parâmetros da função discriminante utilizando as medidas lineares que permitiram classificar as potras quanto à idade e reordená-los nas diferentes

Tabela 11 - Valores médios, desvios-padrão (DP), coeficientes de variação (CV) e parâmetros da função discriminante $\left(\beta_{\mathrm{s}}\right)$ de potros e potras com doze meses de idade, obtidos das medidas angulares

Table 11 - Average, standard deviation (DP), coefficients of variation (CV) and parameters of discriminant function ( $\beta_{s}$ ) of colts and fillies with twelve months age, obtained from angular measures

\begin{tabular}{|c|c|c|c|c|c|c|c|c|}
\hline \multirow[t]{2}{*}{$\begin{array}{l}\text { Variável } \\
\text { Variable }\end{array}$} & \multicolumn{4}{|c|}{$\begin{array}{c}\text { Potro } \\
\text { Colt }\end{array}$} & \multicolumn{4}{|c|}{$\begin{array}{l}\text { Potra } \\
\text { Filly }\end{array}$} \\
\hline & $\beta_{\mathrm{s}}$ & $\begin{array}{c}\text { Média }(\mathrm{cm}) \\
\text { Average }\end{array}$ & $\mathrm{DP}(\mathrm{cm})$ & CV\% & $\beta_{\mathrm{s}}$ & $\begin{array}{c}\text { Média }(\mathrm{cm}) \\
\text { Average }\end{array}$ & $\mathrm{DP}(\mathrm{cm})$ & $\mathrm{CV} \%$ \\
\hline$\overline{\mathrm{AUR}}$ & 10,38009 & $122,2^{\circ}$ & $\pm 3,4^{\circ}$ & 2,8 & 10,19162 & $120,4^{\circ}$ & $\pm 3,4 \mathrm{o}$ & 2,9 \\
\hline ACS & 0,26947 & $24,0^{\circ}$ & $\pm 2,5^{\circ}$ & 10,4 & 0,45286 & $25,10^{\circ}$ & $\pm 3,05$ & 12,1 \\
\hline $\begin{array}{l}\text { Constante } \\
\text { Constant }\end{array}$ & $-638,479$ & - & - & - & $-619,630$ & - & - & - \\
\hline
\end{tabular}

AUR - ângulo úmero-radial; ACS - ângulo coxal-solo.

AUR - humero-radio angle; ACS - coxal-floor angle. 
Tabela 12 - Classificação de potras e potros com doze meses de idade, em função das medidas angulares

Table 12 - Classification of fillies and colts of twelve months age, in function of angular measures

\begin{tabular}{ccc}
\hline $\begin{array}{c}\text { Grupo observado } \\
\text { Observed group }\end{array}$ & \multicolumn{2}{c}{ Após a classificação } \\
& \multicolumn{2}{c}{ Afterclassification } \\
\cline { 2 - 3 } & Potra & Potro \\
Filly & Colt \\
\hline Potra (Filly) & 23 & 7 \\
$\%$ & 74,2 & 36,8 \\
Potro (Colt) & 8 & 12 \\
$\%$ & 25,8 & 63,2 \\
Total & 31 & 19 \\
\hline
\end{tabular}

Percentual de acertos $=68,7 \%$

Correctness percentage $=68.7 \%$ idades podem ser observados na Tabela 13. Estes parâmetros podem ser utilizados na elaboração de função discriminante e avaliação de outras potras da raça Mangalarga Marchador, de acordo com as idades consideradas, identificando os animais sub ou superdesenvolvidos.

A análise discriminante da idade apresentou percentual de acerto igual a $100 \%$ nos primeiros quatro meses de idade, portanto, no período inicial, os animais apresentaram um padrão de desenvolvimento bastante uniforme, provavelmente em razão de a dieta destes animais ter sido basicamente de leite materno (Tabela 14). Aos seis meses de idade, ocorrem os

Tabela 13 - Parâmetros das funções discriminantes de potras, ao nascimento e aos 2, 4, 6, 8, 10 e 12 meses de idade, obtidas com as medidas lineares

Table 13 - Parameters of discriminant functions of fillies at birth and at 2, 4, 6, 8, 10 and 12 months age, obtained by linear measures

\begin{tabular}{|c|c|c|c|c|c|c|c|}
\hline \multirow{2}{*}{$\begin{array}{l}\text { Variável } \\
\text { Variable }\end{array}$} & \multicolumn{7}{|c|}{ Idade $(A g e)$} \\
\hline & 0 & 2 & 4 & 6 & 8 & 10 & 12 \\
\hline$\overline{\text { Constante }\left(\beta_{0}\right)}$ & $-564,6941$ & $-697,1939$ & $-783,1596$ & $-848,0916$ & $-898,8664$ & $-957,0831$ & $-977,4617$ \\
\hline $\mathrm{AC}$ & 0,04256 & $-0,01758$ & $-0,01397$ & 0,55092 & 1,15870 & 1,65035 & 1,87825 \\
\hline $\mathrm{AG}$ & 4,09319 & 4,26339 & 3,87865 & 2,99953 & 2,28052 & 2,14509 & 1,69964 \\
\hline Aços & $-0,95185$ & 0,09285 & 0,96929 & 2,18259 & 2,75450 & 3,35339 & 3,84302 \\
\hline CC & $-1,28204$ & $-0,40182$ & 0,16817 & 0,54380 & 0,94211 & 1,07771 & 1,25219 \\
\hline $\mathrm{CG}$ & 1,24097 & 1,75110 & 2,10201 & 2,25523 & 2,38327 & 2,36201 & 1,91428 \\
\hline $\mathrm{CDL}$ & 1,00455 & 0,82126 & 0,78427 & 0,70900 & 0,70266 & 0,76547 & 0,73547 \\
\hline $\mathrm{CE}$ & 1,49857 & 0,86062 & 0,72712 & 0,40219 & 0,84064 & 0,62980 & 1,36504 \\
\hline $\mathrm{CP}$ & 2,12385 & 2,59718 & 2,96188 & 3,20998 & 3,11548 & 3,20791 & 3,08171 \\
\hline Ccab & 2,57621 & 4,07707 & 4,69879 & 5,13541 & 5,20377 & 5,47151 & 5,04784 \\
\hline LC & 6,83109 & 5,95204 & 4,85548 & 4,22714 & 3,41975 & 3,59400 & 2,43039 \\
\hline LA & $-4,11684$ & $-3,76865$ & $-2,69894$ & $-1,95138$ & $-1,47312$ & $-0,38186$ & 0,49640 \\
\hline LP & $-1,62732$ & $-3,01660$ & $-3,52865$ & $-4,01379$ & $-4,41808$ & $-5,21417$ & $-5,30610$ \\
\hline DEB & $-0,97596$ & $-1,41062$ & $-1,72559$ & $-1,82445$ & $-1,79759$ & $-2,16455$ & $-1,54826$ \\
\hline DCS & 0,13316 & $-0,40052$ & $-0,27210$ & $-0,15632$ & $-0,09334$ & $-0,12527$ & $-0,04108$ \\
\hline DCJ & 4,93687 & 5,15089 & 5,03976 & 4,94690 & 5,43593 & 5,35502 & 4,98657 \\
\hline DjoB & 6,52747 & 5,79055 & 5,02290 & 3,99766 & 3,53034 & 2,45613 & 1,42756 \\
\hline DSJ & 2,88020 & 3,84002 & 4,27476 & 4,96253 & 5,04440 & 5,34004 & 5,40649 \\
\hline DjaB & 1,19081 & 1,76529 & 1,98183 & 2,15258 & 1,86389 & 1,95932 & 2,07694 \\
\hline PT & $-2,80109$ & $-2,28037$ & $-1,97741$ & $-1,88133$ & $-2,00949$ & $-1,85876$ & $-1,74003$ \\
\hline PA & 0,28629 & $-0,04842$ & $-0,49455$ & $-0,65981$ & $-0,90491$ & $-1,19574$ & $-1,24862$ \\
\hline PJ & 20,83895 & 20,88209 & 19,66103 & 17,81977 & 17,52637 & 16,50337 & 15,99859 \\
\hline PB & 3,55303 & 1,94495 & 2,04498 & 2,33016 & 2,19608 & 2,77241 & 2,08421 \\
\hline $\mathrm{PC}$ & 2,11288 & 1,98061 & 1,77861 & 1,76601 & 1,82869 & 0,99133 & 1,85675 \\
\hline $\mathrm{CCA}$ & $-2,19267$ & $-0,35770$ & 1,13881 & 3,14674 & 2,38801 & 3,31093 & 1,14858 \\
\hline $\mathrm{CCP}$ & $-9,09951$ & $-11,05839$ & $-10,61820$ & $-10,62624$ & $-8,85118$ & $-9,93850$ & $-9,02486$ \\
\hline
\end{tabular}

Altura na cernelha - AC; Altura na garupa - AG; Altura do costado - ACos; Comprimento do corpo - CC; Comprimento da garupa - CG; Comprimento do dorso-lombo - CDL; Comprimento da escápula - CE; Comprimento do pescoço - CP; Comprimento da cabeça - Ccab; Largura da cabeça - LC; Largura da anca - LA; Largura do peito - LP; Distância da ponta da escápula ao boleto - DEB; Distância do codilho ao solo - DCS; Distância do codilho ao joelho - DCJ; Distância do joelho ao boleto - DjoB; Distância da soldra ao jarrete - DSJ; Distância do jarrete ao boleto - DJaB; Perímetro torácico - PT; Perímetro do antebraço - PA; Perímetro do joelho - PJ; Perímetro do boleto - PB; Perímetro da canela - PC; Comprimento do casco anterior - CCA; e comprimento do casco posterior - CCP.

$A C$ - Withers heigth; $A G$ - Croup height; $A C$ Cos - chest depth; $C C$ - body length; $C G$ - croup length; $C D L$ - back-loins length; $C E$ - shoulder length; $C P$ - neck length; $C$ cab - head length; $L C$ - head width; $L A$ - hips width; $L P$ - chest width; $D E B$ - distance between shoulder and fetlock; DCS - distance between elbow and floor; DCJ - distance between elbow and knee; DJoB - distance between knee and fetlock; DSJ - distance between stifle and hock; DJaB - distance between hock and fetlock; PT - chest girth; PA - forearm girth; PJ - knee girth; PB - fetlock girth; PC - cannon girth; CCA - hoof length anterior; CCP - hoof length posterior.

\section{R. Bras. Zootec., v.34, n.2, p.600-612, 2005}


Tabela 14 - Resumo da classificação das potras, em função das medidas lineares, ao nascimento e aos 2, 4, 6, 8, 10 e 12 meses de idade

Table 14 - Summary of the classification of fillies, in function of the linear measures at birth and at 2, 4, 6, 8, 10 and 12 months age

\begin{tabular}{cccccccc}
\hline Mês observado & \multicolumn{7}{c}{ Mês estimado } \\
& 0 Estimated month \\
\cline { 2 - 8 } & 2 & 4 & 6 & 8 & 10 & 12 \\
\hline 0 & 41 & 0 & 0 & 0 & 0 & 0 & 0 \\
$\%$ & 100,0 & 0,0 & 0,0 & 0,0 & 0,0 & 0,0 & 0,0 \\
2 & 0 & 41 & 0 & 0 & 0 & 0 & 0 \\
$\%$ & 0,0 & 100,0 & 0,0 & 0,0 & 0,0 & 0,0 & 0,0 \\
4 & 0 & 0 & 39 & 0 & 0 & 0 & 0 \\
$\%$ & 0,0 & 0,0 & 100,0 & 0,0 & 0,0 & 0,0 & 0,0 \\
6 & 0 & 0 & 1 & 33 & 3 & 0 & 0 \\
$\%$ & 0,0 & 0,0 & 2,7 & 89,2 & 8,1 & 0,0 & 0,0 \\
8 & 0 & 0 & 0 & 2 & 25 & 1 & 0 \\
$\%$ & 0,0 & 0,0 & 0,0 & 7,2 & 89,3 & 3,6 & 0,0 \\
10 & 0 & 0 & 0 & 0 & 2 & 17 & 3 \\
$\%$ & 0,0 & 0,0 & 0,0 & 0,0 & 9,1 & 77,3 & 13,7 \\
12 & 0 & 0 & 0 & 0 & 1 & 5 & 25 \\
$\%$ & 0,0 & 0,0 & 0,0 & 0,0 & 3,2 & 16,1 & 80,6 \\
\hline
\end{tabular}

Percentual de acertos $=92,5 \%$.

Correctness percentage $=92.5 \%$.

primeiros erros de classificação, sendo uma potra, das 37 avaliadas, classificada na idade de quatro meses e três potras classificadas na idade de oito meses, erros correspondentes a 2,7 e $8,1 \%$, respectivamente.

Das 28 potras avaliadas aos oito meses de idade, duas potras foram classificadas na idade de seis meses e uma potra classificada na idade de 10 meses, erros que corresponderam a 7,2 e $3,6 \%$, respectivamente. Aos 10 meses de idade, entre as 22 potras avaliadas, duas foram classificadas na idade de oito meses e três potras classificadas na idade de 12 meses, erros que correspondem a 9,1 e $13,7 \%$ nesta idade.

Das 31 potras avaliadas aos 12 meses de idade, uma potra foi classificada na idade de oito meses e cinco classificadas na idade de 10 meses, erros que corresponderam a 3,2 e $16,1 \%$ nesta idade. Estes animais apresentaram claramente desenvolvimento corporal abaixo da média dos animais com 12 meses, portanto, eles poderiam ser direcionados para um programa apropriado de alimentação para tentar recuperá-los antes do término do crescimento ósseo e comprometimento definitivo das características morfológicas.

$\mathrm{Na}$ Tabela 15, podem ser observados os parâmetros das funções discriminantes gerados pelas medidas lineares de potros da raça Mangalarga
Marchador, ao nascimento e aos 2, 4, 6, 8, 10 e 12 meses de idade. Esses parâmetros podem ser utilizados na elaboração das funções discriminantes, a fim de identificar animais fora do padrão de desenvolvimento conforme a idade.

A classificação dos potros em função da análise discriminante utilizando as medidas lineares permitiram classificá-los quanto à idade e reordená-los nas diferentes idades (Tabela 16). Até o segundo mês de idade, a análise apresentou percentual de acerto de $100 \%$, que no quarto mês foi de $97,7 \%$. Estes resultados foram semelhantes aos observados nas potras, exceto a ocorrência de um potro com 4 meses de idade, que foi classificado no grupo de seis meses de idade, portanto, na fase de pré-desmame, os potros apresentaram desenvolvimento uniforme.

Assim como nas potras, os primeiros erros mais significativos na classificação dos potros ocorreram aos seis meses de idade, quando seis dos 35 potros avaliados foram classificados no grupo de oito meses de idade, erro de $17,1 \%$, e um potro foi classificado na idade de 4 meses, erro igual a 2,9\%, enquanto aos oito meses de idade três dos 29 potros avaliados foram classificados na idade de seis meses, erro que correspondeu a $10,3 \%$.

Aos dez meses de idade pode ser observado que dos 20 potros avaliados, quatro foram classificados na idade de oito meses e outros quatros, na idade de 12 meses, erros que corresponderam a 20\% do grupo, enquanto aos 12 meses de idade, dos 19 avaliados, um potro classificado na idade de oito meses e outros quatro, na idade de 10 meses, erros que corresponderam a 5,3 e 21,0\% nesta idade.

Os erros de classificação que ocorreram após o desmame, tanto nas potras quanto nos potros, portanto, estão provavelmente relacionados às práticas de manejo pós-desmame inerentes a cada haras, como qualidade e quantidade da dieta e/ou problemas sanitários.

Em relação à análise discriminante das medidas angulares dos potros e das potras, em função da idade, não houve formação de grupos claramente definidos por faixas etárias. O percentual de acerto médio foi de $55,2 \%$ nas potras e de $52 \%$ nos potros; portanto, as medidas lineares são de maior eficácia para discriminar a idade de potros e potras.

Segundo Thomassian (1997), os eqüinos atingem a maturidade óssea entre o 2o e $3 \underline{o}$ ano de vida, quando é observada a calcificação das regiões fisárias dos ossos, ou seja, a fusão da diáfise com a epífise. $\mathrm{Na}$ 
Tabela 15 - Parâmetros das funções discriminantes de potros, ao nascimento e aos 2, 4, 6, 8, 10 e 12 meses de idade, obtidas com as medidas lineares

Table 15 - Parameters of discriminant functions of colts at birth and at 2, 4, 6, 8, 10 and 12 months age, obtained by linear measures

\begin{tabular}{|c|c|c|c|c|c|c|c|}
\hline \multirow{2}{*}{$\begin{array}{l}\text { Variável } \\
\text { Variable }\end{array}$} & \multicolumn{7}{|c|}{ Idade $(A g e)$} \\
\hline & 0 & 2 & 4 & 6 & 8 & 10 & 12 \\
\hline Constant & $-775,130$ & $-954,949$ & -1095 & -1188 & -1256 & -1316 & -1351 \\
\hline $\mathrm{AC}$ & 0,24605 & 0,33042 & 0,34033 & 0,43608 & 0,62069 & 0,71425 & 0,90119 \\
\hline $\mathrm{AG}$ & 2,73253 & 3,02058 & 2,90689 & 2,57489 & 2,46357 & 2,29805 & 2,38579 \\
\hline ACOS & $-10,2036$ & $-9,93328$ & $-9,55339$ & $-8,61664$ & $-8,40787$ & $-7,78795$ & $-7,86789$ \\
\hline $\mathrm{CC}$ & $-0,68930$ & $-0,02622$ & 0,36179 & 0,63086 & 0,78746 & 0,95673 & 1,00381 \\
\hline $\mathrm{CG}$ & 2,74701 & 3,40199 & 3,96906 & 4,29183 & 4,68279 & 4,61381 & 4,36099 \\
\hline CDL & 0,31940 & 0,33473 & 0,44636 & 0,19672 & 0,17254 & 0,43207 & 0,39363 \\
\hline $\mathrm{CE}$ & 1,13877 & 0,22747 & 0,08321 & 0,15776 & 0,33750 & 0,77256 & 1,53412 \\
\hline $\mathrm{CP}$ & 0,43546 & 0,65821 & 0,86619 & 1,18688 & 1,03375 & 0,95638 & 0,90066 \\
\hline Ccab & 6,83509 & 8,44684 & 9,57744 & 10,13122 & 10,75619 & 10,71101 & 10,90857 \\
\hline LC & 3,18229 & 1,89756 & 1,95637 & 1,12749 & 0,69981 & 0,38424 & 0,25467 \\
\hline LA & 0,54055 & 0,51651 & 0,74513 & 0,80614 & 0,96176 & 1,31325 & 1,45538 \\
\hline LP & 0,28785 & 0,09895 & $-0,15711$ & $-0,37597$ & $-0,96538$ & $-1,15658$ & $-1,60837$ \\
\hline DEB & 3,31059 & 3,35808 & 2,91593 & 3,17398 & 3,13492 & 3,16934 & 3,44560 \\
\hline DCS & $-0,89628$ & $-1,08780$ & $-1,24794$ & $-1,34214$ & $-1,39526$ & $-1,40519$ & $-1,36660$ \\
\hline DCJ & 6,81319 & 6,32468 & 6,31534 & 6,32905 & 6,88552 & 7,24794 & 6,66577 \\
\hline DjoB & 9,27755 & 8,68597 & 8,70523 & 8,42518 & 8,27473 & 8,31708 & 7,59586 \\
\hline DSJ & 4,66541 & 5,53680 & 6,00815 & 6,48418 & 6,69477 & 6,54119 & 6,81626 \\
\hline DjaB & 1,64148 & 1,54528 & 1,43838 & 1,39324 & 1,14628 & 1,15389 & 0,75441 \\
\hline PT & $-0,97797$ & $-0,33533$ & 0,08831 & 0,07030 & 0,23823 & 0,25062 & 0,28198 \\
\hline PA & $-2,19604$ & $-1,57146$ & $-0,86513$ & $-0,79348$ & $-0,56149$ & $-0,60253$ & $-0,71729$ \\
\hline PJ & 24,63351 & 25,78709 & 25,60059 & 24,57166 & 23,51601 & 22,63092 & 23,23590 \\
\hline PB & 7,70482 & 4,16564 & 2,77848 & 2,46928 & 2,21903 & 1,98104 & 2,36132 \\
\hline $\mathrm{PC}$ & $-6,42764$ & $-6,78590$ & $-7,27355$ & $-7,06034$ & $-7,14584$ & $-6,72198$ & $-8,24687$ \\
\hline $\mathrm{CCA}$ & 6,64540 & 10,34134 & 12,81130 & 16,28545 & 14,38457 & 13,70361 & 14,41873 \\
\hline $\mathrm{CCP}$ & $-13,2559$ & $-11,9381$ & $-10,1712$ & $-9,50291$ & $-7,66478$ & $-5,60777$ & $-6,07536$ \\
\hline
\end{tabular}

Altura na cernelha - AC; Altura na garupa - AG; Altura do costado - ACos; Comprimento do corpo - CC; Comprimento da garupa - CG; Comprimento do dorso-lombo - CDL; Comprimento da escápula - CE; Comprimento do pescoço - CP; Comprimento da cabeça - Ccab; Largura da cabeça - LC; Largura da anca - LA; Largura do peito - LP; Distância da ponta da escápula ao boleto - DEB; Distância do codilho ao solo - DCS; Distância do codilho ao joelho - DCJ; Distância do joelho ao boleto - DjoB; Distância da soldra ao jarrete - DSJ; Distância do jarrete ao boleto - DJaB; Perímetro torácico - PT; Perímetro do antebraço - PA; Perímetro do joelho - PJ; Perímetro do boleto - PB; Perímetro da canela - PC; Comprimento do casco anterior - CCA; e comprimento do casco posterior - CCP.

$A C$ - Withers heigth; $A G$ - Croup height; $A C$ Cos - chest depth; $C C$ - body length; $C G$ - croup length; $C D L$ - back-loins length; $C E$ - shoulder length; $C P$ - neck length; Ccab - head length; $L C$ - head width; $L A$ - hips width; $L P$ - chest width; DEB - distance between shoulder and fetlock; DCS - distance between elbow and floor; DCJ - distance between elbow and knee; DJoB - distance between knee and fetlock; DSJ - distance between stifle and hock; DJaB - distance between hock and fetlock; PT - chest girth; PA - forearm girth; PJ - knee girth; PB - fetlock girth; PC - cannon girth; CCA - hoof length anterior; CCP - hoof length posterior.

raça Mangalarga Marchador, os animais são avaliados para efeito de registro definitivo aos 36 meses de idade, portanto, ao avaliar as medidas morfométricas no primeiro ano de vida, possibilitaria aos criadores e técnicos a oportunidade de correção de problemas referentes a animais sub ou super-desenvolvidos.

Os animais sub ou superdesenvolvidos devem ser submetidos a novos programas de manejo nutricional e ou sanitário, visando corrigir seu desenvolvimento, para obter registro definitivo na ABCCMM e prevenir distúrbios ósteos-articulares, como as epifisites, decorrentes de dietas inadequadas (Rezende et al., 2000a).
Nos animais subdesenvolvidos, as alterações no manejo nutricional devem considerar a possibilidade de ocorrência das deformidades flexurais dos membros (Meyer, 1995), de forma mais evidente quando há fornecimento de dietas ad libitum, conforme observado por Cymbaluk (1989). Segundo Rezende et al. (2000a), a suplementação alimentar com ração concentrada e sal mineral, limitada a $1,5 \%$ do peso vivo, de potros recém-desmamados da raça Mangalarga Marchador, propiciou ganho compensatório na medida da altura na cernelha sem a ocorrência de deformidades flexurais nos membros. 
Tabela 16 - Resumo da classificação dos potros, em função das medidas lineares, ao nascimento e aos 2, 4, 6, 8, 10 e 12 meses de idade Table 16 - Summary of classification of colts, in function of the linear measures at birth and at 2, 4, 6, 8, 10 and 12 months age

\begin{tabular}{cccccccc}
\hline Mês observado & \multicolumn{7}{c}{$\begin{array}{c}\text { Mês estimado } \\
\text { Estimated month }\end{array}$} \\
\cline { 2 - 8 } & 0 & 2 & 4 & 6 & 8 & 10 & 12 \\
\hline 0 & 55 & 0 & 0 & 0 & 0 & 0 & 0 \\
$\%$ & 100,0 & 0,0 & 0,0 & 0,0 & 0,0 & 0,0 & 0,0 \\
2 & 0 & 52 & 0 & 0 & 0 & 0 & 0 \\
$\%$ & 0,0 & 100,0 & 0,0 & 0,0 & 0,0 & 0,0 & 0,0 \\
4 & 0 & 0 & 43 & 1 & 0 & 0 & 0 \\
$\%$ & 0,0 & 0,0 & 97,7 & 2,3 & 0,0 & 0,0 & 0,0 \\
6 & 0 & 0 & 1 & 28 & 6 & 0 & 0 \\
$\%$ & 0,0 & 0,0 & 2,9 & 80,0 & 17,1 & 0,0 & 0,0 \\
8 & 0 & 0 & 0 & 3 & 26 & 0 & 0 \\
$\%$ & 0,0 & 0,0 & 0,0 & 10,3 & 89,7 & 0,0 & 0,0 \\
10 & 0 & 0 & 0 & 0 & 4 & 12 & 4 \\
$\%$ & 0,0 & 0,0 & 0,0 & 0,0 & 20,0 & 60,0 & 20,0 \\
12 & 0 & 0 & 0 & 0 & 1 & 4 & 14 \\
$\%$ & 0,0 & 0,0 & 0,0 & 0,0 & 5,3 & 21,0 & 73,7 \\
\hline
\end{tabular}

Percentual de acertos $=90,5 \%$.

Correctness percentage $=90.5 \%$.

\section{Conclusões}

Os machos são, em geral, maiores que as fêmeas nas medidas lineares que variam em função do sexo, nas diferentes idades avaliadas, exceto a medida de perímetro torácico, que apresentou valor médio maior nas potras.

As medidas lineares são mais interessantes na identificação de animais sub ou superdesenvolvidos, pois permitem maiores percentuais de acerto na classificação dos animais nas faixas etárias avaliadas até os 12 meses de idade.

\section{Agradecimento}

À Fundação de Amparo a Pesquisa do Estado do Rio de Janeiro, à Coordenação de Aperfeiçoamento de Pessoal de Nível Superior e à Associação Brasileira de Criadores do Cavalo Mangalarga Marchador, pelo apoio financeiro.

\section{Literatura Citada}

ALMEIDA, F.Q.; BRITO, C.O.; SANTOS, E.M. et al. Crescimento de potros da raça Mangalarga Marchador. In: REUNIÃO ANUAL DA SOCIEDADE BRASILEIRA DE ZOOTECNIA, 37., 2000, Viçosa, MG. Anais... Viçosa, MG: Sociedade Brasileira de Zootecnia, p.1-3. 2000.
ANDRADE L.S. Efeito da conformação sobre o tipo e eficiência da marcha. In: SIMPÓSIO NORDESTINO DO CAVALO MANGALARGA MARCHADOR-ANDAMENTO, 1., 1986, Recife. Anais... Recife: Pernambuco. p.31-34. 1986.

ÁRNASON, T. Genetic studies on conformation and performance of Icelandic Toelter Horses. I - Estimation of non-genetic effects and genetic parameters. Acta Agricultural Scandinavia, v.34, p.409-427, 1984.

ASSOCIAÇÃO BRASILEIRA DOS CRIADORES DO CAVALO MANGALARGA MARCHADOR - ABCCMM. Estatuto. Belo Horizonte: O Lutador, 1992. 89p.

BARBOSA, C.G. Estudo morfométrico na raça Mangalarga Marchador. Uma abordagem multivariada. Belo Horizonte: Universidade Federal de Minas Gerais, 1993. 76p. Dissertação (Mestrado em Zootecnia) - Universidade Federal de Minas Gerais, 1993.

BREIER, B.H.; GLUCKMAN, P.D. The regulation of postnatal growth: Nutritional influences on endocrine pathways and function of the somatotropic axis. Livestock Production Science, v.27, p.77-94, 1991.

BREIER, B.H.; OLIVER, M.H.; GALLEAR, B.W. Regulation of growth and metabolism during postnatal development. In: CRONJÉ, P.B. (Ed.) Ruminant physiology: digestion, metabolism, growth and reproduction. Reading: CAB International, 2000. p.187-204.

BRITO, A.A. Avaliação e mensuração. Curso de capacitação de técnicos e árbitros da raça Mangalarga Marchador. Belo Horizonte: ABCCMM, 1990. 132p.

CABRAL, G.C. Avaliação morfométrica e estudo das curvas de crescimento de eqüinos da raça Mangalarga Marchador. Seropédica: Universidade Federal Rural do Rio de Janeiro, 2002. 109p. Dissertação (Mestrado em Zootecnia) Universidade Federal Rural do Rio de Janeiro, 2002.

CABRAL, G.C.; ALMEIDA, F.Q.; AZEVEDO, P.C.N. et al. Avaliação morfométrica de eqüinos da raça Mangalarga Marchador: medidas angulares. Revista Brasileira de Zootecnia, v.33, n.6, p.1790-1797, 2004a.

CABRAL, G.C.; ALMEIDA, F.Q.; QUIRINO, C.R. et al. Avaliação morfométrica de eqüinos da raça Mangalarga Marchador: índices de conformação e proporções corporais. Revista Brasileira de Zootecnia, v. 33, n.6, p.1798-1805, 2004b.

CABRERA, L.C.; FERNANDES, L.C.O.; MORAES, C.M.M. Composição de leite de éguas P.S.I. e desenvolvimento ponderal de sua crias. A Hora Veterinária. n.55, p.24-29, 1990.

CAMARGO, M.X.; CHIEFFI, A. Ezoognósia. São Paulo: CPA/ Intituto de Zootecnia, 1971. 320p

CARNEIRO, G.G.; FONTES, L.R.; PEREIRA, H.C. Informações preliminares sobre o cavalo Mangalarga Marchador. Arquivos da Escola Superior de Veterinária da UREMG, v.5, p.5-9, 1952.

CYMBALUCK, N.F. Effects of dietary energy source and level of feed intake on growth of weanling horses. Equine Practice, v.11, n.9, p.29-33, 1989.

FARIA, R. Avaliação genética e fenotípica de características de conformação em potros das raças eqüinas de sela e proposição de índice de seleção. 1998. 134p. Dissertação (Mestrado em Zootecnia) - Universidade Federal de Viçosa, Viçosa, 1998.

GARCÍA, F.F.; GARCÍA, M.H.; MACARRO, J.B.A. et al. Morfoestructura del caballo Árabe en España. Archivos de Zootecnia, v.36, n.136, p.269-277, 1987.

GATFORD, K.L.; EGAN, A.R.; CLARK, I.J. et al. Sexual dimorphism of the somatotropic axis. Journal of Endocrinology, v. 157, p. 501-508, 1998.

R. Bras. Zootec., v.34, n.2, p.600-612, 2005 
GREEN, D.A. Study of growth rate in thoroughbred foals. British Veterinary Journal, v.125, p.539-545, 1969.

HOLMSTRÖM, M.; MAGNUSSON, L.E.; PHILIPSSON, J. Variation in conformations of Swedish Warmblood horses and conformational characteristics of elite sport horses. Equine Veterinary Journal, v.22, n.3, p.186-193. 1990.

JAINUDEE, M.R.; HAFEZ, E.S.E. Gestação, fisiologia prénatal e parto. In: HAFEZ, E.S.E. (Ed.) Reprodução animal. 4 ed. São Paulo: Manole, 1982. p. 280-321.

KAHTTREE, R.; NAIK, D.N. Multivariate data reduction and discrimination with $\mathbf{S A S}{ }^{\circledR}$ software. North Carolina: SAS Institute Inc. 2000. 559p.

LAGE, M.C.G.R. Caracterização morfométrica, dos aprumos e do padrão de deslocamento de eqüinos da raça Mangalarga Marchador e suas associações com a qualidade da marcha. Belo Horizonte: Universidade Federal de Minas Gerais, 2001. 114p. Tese (Doutorado em Ciência Animal) - Universidade Federal de Minas Gerais, 2001.

MANSO FILHO, H.C.; COSTA, H.E.C.; MANSO, H.C. Desenvolvimento corporal de potros Mangalarga Marchador durante a fase de amamentação. In: REUNIÃO ANUAL DA SOCIEDADE BRASILEIRA DE ZOOTECNIA, 35., 1998, Botucatu. Anais... Botucatu: Sociedade Brasileira de Zootecnia, 1998. p.662-664.

MEYER, H. Alimentação de cavalos. São Paulo: Varela, 1995. $303 p$.

MOLINA, A.; VALERA, M.; SANTOS, R. et al. Genetic parameters of morphofunctional traits in Andalusian horse. Livestock Production Science, v.60, p.295-303. 1999.

NASCIMENTO, J.F. Mangalarga Marchador: tratado morfofuncional. Belo Horizonte: Associação Brasileira dos Criadores do Cavalo Mangalarga Marchador, 1999. 577p.

PINTO, L.F.B. Análise multivariada das medidas morfométricas de eqüinos da raça Mangalarga Marchador. Seropédica: Universidade Federal Rural do Rio de Janeiro, 2003. 116p. Dissertação (Mestrado em Zootecnia) - Universidade Federal Rural do Rio de Janeiro, 2003.

REZENDE, A.S.C.; SAMPAIO, I.B.M.; LEGORRETA, G.L. et al. Effect of two different nutritional programs on orthopedic alterations in Mangalarga Marchador foals. Journal of Equine Veterinary Science, v.20, n.10, p.651-656, 2000a.
REZENDE, A.S.C.; SAMPAIO, I.B.M.; LEGORRETA, G.L. et al. Efeito de dois diferentes programas nutricionais sobre o desenvolvimento corporal de potros Mangalarga Marchador. Revista Brasileira de Zootecnia, v.29, n.2, p.495-501, $2000 \mathrm{~b}$.

RIBEIRO, D.B. O cavalo: raças, qualidade e defeitos. Rio de Janeiro: Globo: 1988. 318p.

SAASTAMOINEN, M.T. Factors affecting growth and development of foals and young horses. Acta Agriculture Scandinavica, v.40, p.387-396, 1990.

STATISTICAL ANALYSIS SYSTEM - SAS. STAT ${ }^{\circledR}$ user's guide. Cary: 1996.

THOMASSIAN A. Enfermidades dos cavalos. São Paulo: Varela, 1997. 643p.

THOMPSON, K.N. Skeletal growth rates of weanling and yearling Thoroughbred horses. Journal of Animal Science, v.73, p.2513-2517, 1995.

TOLEDO, A.P. Mecânica de sustentação e locomoção dos eqüinos. Guarulhos: Parma, 1985. 177p.

TORRES, A.P.; JARDIM, W.R. Criação do cavalo e de outros eqüinos. São Paulo: Nobel, 1992. 654p.

ZAMBORLINI, L.C.; BERGMANN, J.A.G.; PEREIRA, C.S. et al. Estudo genético quantitativo das medidas lineares da raça Mangalarga Marchador. I. Estimativas dos fatores de ambiente e parâmetros genéticos. Revista Brasileira de Ciências Veterinárias, v.3, n.2, p.33-37, 1996.

ZAMBORLINI, L.C. Estudo genético quantitativo das medidas lineares da raça Mangalarga Marchador. Belo Horizonte: Universidade Federal de Minas Gerais, 1996. 47p. Tese (Doutorado em Ciência Animal) - Universidade Federal de Minas Gerais, 1996.

ZECHNER, P.; ZOHMAN, F.; SÖLKNER, J. et al. Morphological description of the Lipizzan horse population. Livestock Production Science, v.69, p.163-177, 2001. 